\title{
Implementing Dosimetry in GATE: Dose-Point Kernel Validation with GEANT4 4.8.1
}

\author{
Ludovic Ferrer, ${ }^{1,2}$ Nicolas Chouin, ${ }^{1,3}$ Abdalkader Bitar, ${ }^{1,3}$ Albert Lisbona, ${ }^{1,2}$ \\ and Manuel Bardiès ${ }^{1,3}$ \\ ${ }^{1}$ INSERM, U601, Nantes, France \\ ${ }^{2}$ Medical Physics Department, CRLCC Rene Gauducheau, Saint-Herblain, France \\ ${ }^{3}$ Université de Nantes, Faculté de Médecine, Nantes, France
}

\begin{abstract}
GATE is a recent Monte Carlo code, based on GEANT4, and used in nuclear medicine mainly for imaging and detector design. Our goal was to implement dosimetry within GATE (i.e., combining the excellent potential of Gate for image modeling with GEANT4 dosimetric capabilities. The latest release of GEANT4 (4.8.1) completely revised the electron multiple scattering propagation algorithm. In this work, we calculated dose point kernels (DPK) for 0.01, 0.05, 0.1, 1, and $3 \mathrm{MeV}$ monoenergetic electrons. We then compared our results with data obtained with another Monte Carlo code (MCNPX) or from the reference publication from Berger and Seltzer. To facilitate comparison, all calculated dose distributions were scaled to the corresponding $R_{C S D A}$, as given by the ESTAR NIST web database. Some GEANT4 parameters (i.e., Stepmax), or the shell thickness, had to be adjusted in order to achieve good agreement for energies below $1 \mathrm{MeV}$. For all energies except $10 \mathrm{keV}$, calculated DPKs do not differ significantly from the reference, as assessed by a Kolmogorov-Smirnov test. This preliminary step allowed us to consider the integration of GEANT4 dosimetric capabilities within the Gate framework.
\end{abstract}

Key words: Monte Carlo simulation, dosimetry, radiotherapy

\section{INTRODUCTION}

GATE (GEANT4-based Application for Emission Tomography) is a recent Monte Carlo (MC) code used in nuclear medicine mainly for imaging and detector design purposes. ${ }^{1}$ GATE is based on GEANT4 (Geometry and Tracking), a general MC simulation toolkit. ${ }^{2}$ GEANT4-derived codes have been evaluated to give dosimetry of external beam radiation in the context of radiotherapy, but to our knowledge, none have been used for nuclear medicine dosimetry. ${ }^{3,4}$ Our

Address reprint requests to: Ludovic Ferrar, Medical Physics Department, RLCC Rene Gauducheau, Boulevard, J. Monod, 44805 St. Herblain, France

E-mail: l-ferrer@nantes.fnclcc.fr aim in this study was to implement dosimetry within GATE (i.e., combining the excellent potential of GATE for imaging modeling with GEANT4 dosimetric capabilities). It was first necessary to assess GEANT4 dosimetric potential for both electrons and photons at the energies encountered in nuclear medicine. As the latest release of GEANT4 (version 8.1) completely revised the electron multiple scattering Coulomb (MSC) propagation algorithm, validations on electron transportation and interactions had to be performed..$^{5}$ We compared dose point kernels (DPKs) for monoenergetic electrons calculated with GEANT4 and MCNPX, another generalpurpose radiation transport MC code. ${ }^{6} \mathrm{DPKs}$ calculated with GEANT4 were further compared to reference data published in 1991 by Seltzer using ETRAN. ${ }^{7}$ 


\section{MATERIALS AND METHODS}

DPKs correspond to the energy deposited at a distance from a point source in water-equivalent spherical shells. In MC calculations, a simulation of particles crossing interfaces between two media is often very time consuming. However, DPKs are calculated for particles propagating in uniform media; therefore, energy scoring can be performed in spherical shells, but propagation is carried out throughout homogeneous medium. Therefore, a way to speed up calculations was to consider a single sphere of water as a propagating medium, with a radius superior to the maximum range of the considered radiation.

To facilitate comparison, all calculated dose distributions were scaled to the corresponding $\mathrm{R}_{\text {CSDA }}$, as given by the ESTAR NIST web database. ${ }^{8} \mathrm{R}_{\mathrm{CSDA}}$ reflects the distance traveled by electrons of a given energy down to rest, if the energy-loss rate along the track is equal to the mean energy-loss rate (i.e., under the continuous slowing down approximation).

\section{Monte Carlo Codes}

GEANT4 and MCNPX are both general-purpose radiation transport MC codes. MCNPX is a major extension of the MCNP code. ${ }^{9}$ Although out of the scope of this work, it is worth mentioning that MCNP and MCNPX on one hand, and GEANT4 on the other hand, implement different transport and interaction algorithms.

The step-size of the electron is a very important parameter to consider when simulating electron transportation and interaction in matter. This is especially relevant when the considered scor-

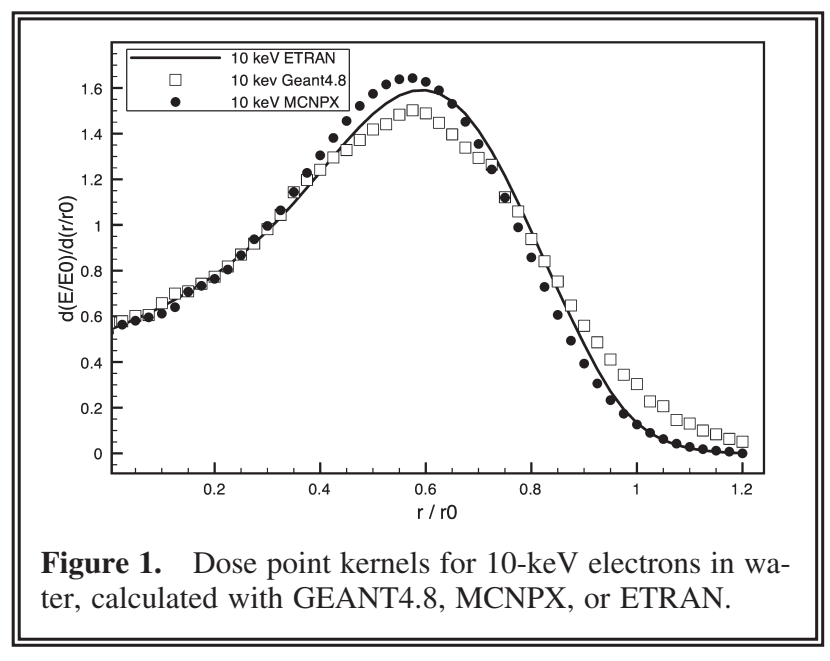

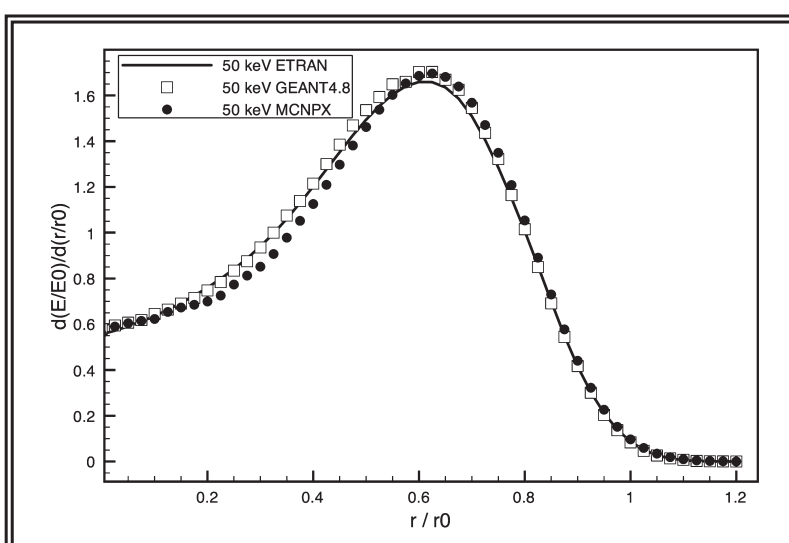

Figure 2. Dose point kernels for $50-\mathrm{keV}$ electrons in water, calculated with GEANT4.8, MCNPX, or ETRAN.

ing layers are thin, as compared to electron stepsize. Seltzer and other authors sampled energy scoring over rather thin layers equal to 0.025

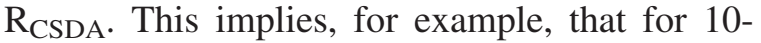
$\mathrm{keV}$ electrons, the shell thickness corresponds to $0.06 \mu \mathrm{m}$. The default electron step-sizes of both $\mathrm{MC}$ codes are of the same order of magnitude (i.e., at the limit of validity of the MSC algorithm). It is, therefore, necessary to tune the electron step-size for low energies. GEANT4 and MCNPX are using different approaches to set the electron step-size.

\section{GEANT4 4.8.1}

GEANT4 comes with several sets of physics processes, interaction models, and cross-section tables, referred to as "Standard," "Low-Energy," and "Penelope" packages. Users have to choose

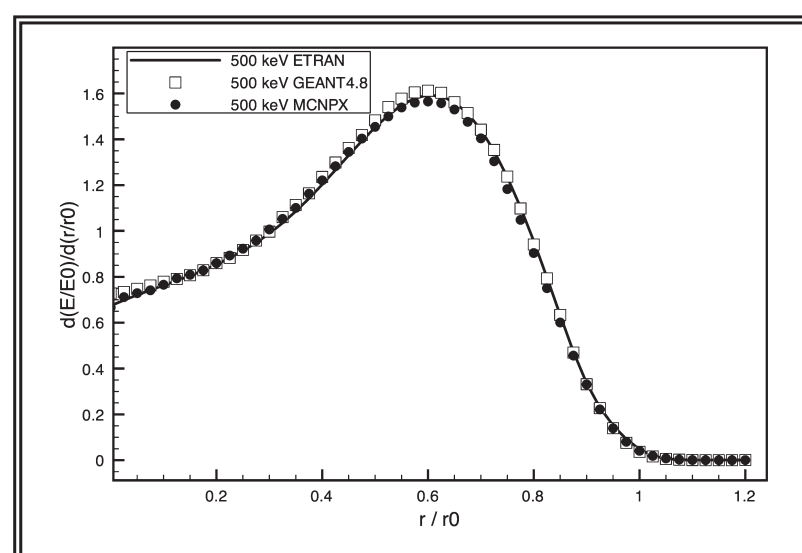

Figure 3. Dose point kernels for $500-\mathrm{keV}$ electrons in water, calculated with GEANT4.8, MCNPX, or ETRAN. 
the package that will be used during the simulations. According to GEANT4 documentation, the standard package model is relevant down to 1 $\mathrm{keV}$ (i.e., an energy threshold compatible with nuclear medicine dosimetric calculation). DPK calculations were carried out by simulating 200,000 particles for all considered energies. Secondary production cut-off was set to $1 \mathrm{~mm}$ (i.e., delta-rays were produced only if their stopping range in water was superior to that value). Below this threshold, the energy loss was, therefore, modeled under the continuous slowing down approximation. GEANT4 allows several means to control the particle step-size. ${ }^{10}$ One of them consists of using a dedicated $\mathrm{C}++$ class responsible of limiting the step-size by a user-given value, STEPMAX. In our experiment, deposited energy was scored into bins equivalent to the shells' thicknesses. In order to force electrons to interact at least once in a given bin, STEPMAX was set equal to the bin width. By default, energy is deposited at the endpoint of the step. A specific class, called UserSteppingAction, was used to assign the deposited energy to a random location along the step length.

\section{MCNPX 2.5.0}

Energy deposits were tallied on a mesh superimposed on the uniform water sphere mentioned above, as multiple boundary crossing may impact electron transport accuracy at low energy. ${ }^{11}$ That option is called Spherical Mesh Tally in MCNPX, 2.5.0 (equivalent to the +F6 tally). As recommended by Schaart et al. and Reynaert et al., we used the ITS energy indexing algorithm. ${ }^{11,12} \mathrm{We}$ simulated 1 million particles for $0.01-, 0.05-$,

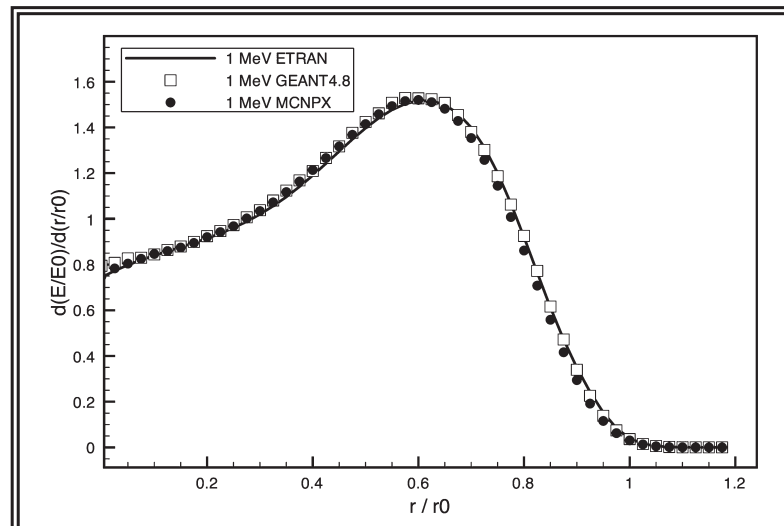

Figure 4. Dose point kernels for $1-\mathrm{MeV}$ electrons in water, calculated with GEANT4.8, MCNPX, or ETRAN.

\begin{tabular}{|lcr||}
\hline $\begin{array}{l}\text { Table 1. Statistical Comparison of Dose Point Kernel } \\
\text { Calculated by GEANT } 4.8 \text { with Results Published by } \\
\text { Seltzer }\end{array}$ \\
\hline Energy $(\mathrm{MeV})$ & $D$ & p-value \\
\hline 0.01 & 0.1224 & 0.832 \\
0.05 & 0.0812 & 0.995 \\
0.10 & 0.1020 & 0.950 \\
0.50 & 0.0816 & 0.995 \\
1.00 & 0.0816 & 0.995 \\
3.00 & 0.0816 & 0.995 \\
\hline
\end{tabular}

$0.1-$, and $1-\mathrm{MeV}$ monoenergetic electrons. The statistical uncertainty associated to each tally was less than $3 \%$ for all energies. MCNPX is a class I MC code, ${ }^{13}$ so all precalculated and tabulated data for electrons are stored in an energy grid. That energy grid corresponds to the energy step that characterizes the electron's condensed, random walk. An energy step of path length $s$ is divided into $m$ substeps, each of path length $s / m$, to model more accurately the electron's trajectory. Angular scattering and secondary particle production (knock-on electrons and Bremsstrahlung photons) are calculated at the level of each substep. It is advised that an electron should experience at least 10 substeps in any material of importance to the transport problem. To verify this condition, we divided the energy steps in 70 substeps (ESTEP $=$ 70) for all energies.

\section{STATISTICAL TESTS}

To compare GEANT4 calculated DPKs to MCNPX or published DPKs, a Kolmogorov-Smirnov (KS) statistical test was used. ${ }^{14}$ This test determines if two independent samples have been drawn from the same population. It doesn't make any assumption regarding the distribution of the data. The principle of this test is to determine the cumulative fraction function of the two distributions and calculate $D$, the maximum vertical de-

Table 2. Statistical Comparison of Dose Point Kernels Calculated by GEANT 4.8 and MCNPX

\begin{tabular}{lcr}
\hline Energy $(\mathrm{MeV})$ & $D$ & $\mathrm{p}$-value \\
\hline 0.01 & 0.1667 & 0.474 \\
0.05 & 0.0417 & 0.999 \\
0.10 & 0.0833 & 0.995 \\
0.50 & 0.0833 & 0.995 \\
1.00 & 0.0625 & 0.999 \\
\hline
\end{tabular}


Table 3. Statistical Comparison of Dose Point Kernel Calculated by MCNPX with Results Published by Seltzer $^{7}$

\begin{tabular}{lcc}
\hline Energy $(\mathrm{MeV})$ & $D$ & p-value \\
\hline 0.01 & 0.0838 & 0.994 \\
0.05 & 0.0625 & 1.000 \\
0.10 & 0.0670 & 1.000 \\
0.50 & 0.0625 & 1.000 \\
1.00 & 0.0625 & 1.000 \\
\hline
\end{tabular}

viation. If $D$ is small (i.e., if the two sample cumulative distributions do not differ much), this suggests that the samples come from the same population. We used $R$, a free software environment for statistical computing and graphics (www.R-project.org) to perform all statistical tests.

\section{RESULTS}

DPKs calculated with GEANT4 and MCNPX were compared to the results published by Seltzer (obtained with ETRAN) at 0.01, 0.05, 0.1, 0.5, and $1 \mathrm{MeV}$. In addition, GEANT4 and ETRAN DPKs were compared for $3 \mathrm{MeV}$ electrons. Figures 1-4 represent DPKs obtained at 10, 50, and $500 \mathrm{keV}$, and $1 \mathrm{MeV}$, respectively. As can be seen, the three compared DPK distributions agree well, except at $10 \mathrm{keV}$.

DPK distributions were compared using a KS statistical test. Table 1 shows the results of the statistical test comparing GEANT 4.8.1 versus ETRAN DPKs. The maximum distance between cumulative fraction plot, $D$, is less than or equal to 0.10 ( $p>0.95$ ), thus suggesting that both distributions come from the same population, except at $10 \mathrm{keV}$. Table 2 shows the results of the statistical test comparing GEANT 4.8.1 versus MCNPX DPKs. Here again, the agreement between the two distributions is good, except at $10 \mathrm{keV}$. Table 3 shows the results of the statistical test comparing MCNPX versus ETRAN DPKs. The agreement is excellent at all energies, which is not surprising, as MCNP (and MCNPX) implemented ETRAN algorithms for electron transport. We observed that increasing the spherical layer thicknesses results in a better agreement between MCNPX and ETRAN results (data not shown). If one intends to perform cellular dosimetry studies with MCNPX, these problems should be studied in detail.

\section{DISCUSSION AND CONCLUSIONS}

In this work, we calculated DPKs for monoenergetic electrons using the latest release of GEANT4 (4.8.1). We compared GEANT4-based DPKs against published DPKs as well as DPKs calculated in our laboratory with MCNPX 2.5.0. Comparison between GEANT4 and published DPKs gives good results, except for $10-\mathrm{keV}$ electrons. This may indicate that the limits of the "standard" algorithm implemented within GEANT4 (4.8.1) were reached. The scoring of energy deposits in very thin regions (approximately or less than $1 \mu \mathrm{m}$ ) seems questionable. However, this is unlikely to impact significantly dose calculations performed at the organ scale for conventional nuclear medicine isotope dosimetry. The range of $10-\mathrm{keV}$ electrons in water is approximately $2.5 \mu \mathrm{m}$ (i.e., several orders of magnitude less than the spatial resolution of currently available imaging systems, including small-animal imaging systems). If one is interested in cellular dosimetry, further investigations should be performed with the "Low Energy" or "Penelope" packages available in GEANT4. For nuclear medicine dosimetric calculations involving patients or small animals, the standard package should be considered as acceptable.

In future work, photon DPKs will also be calculated, as this may provide the determination of the delivered dose at the organ/patient scale. This would allow for the integration of dosimetry within Gate.

\section{ACKNOWLEDGMENTS}

This work was partly funded by the Atomic Energy Commission of Syria (Abdalkader Bitar), the "Comité de Loire-Atlantique de la Ligue Nationale de Lutte Contre le Cancer" (NC), and the ACI 2004 "Cancéropôles en émergeance."

\section{REFERENCES}

1. Jan S, Santin G, Strul D, et al. GATE: A simulation toolkit for PET and SPECT. Phys Med Biol 2004;49: 4543. Online document at: http://opengatecollaboration.healthgrid.org Accessed March 9, 2007.

2. Agostinelli S, Allison J, Amako K, et al. GEANT4-a simulation toolkit. Nucl Instrum Meth Phys Res A 2003;506:250. Online document at: http://geant4.web. cern.ch/geant4 Accessed March 9, 2007. 
3. Ballester F. Monte Carlo dosimetric study of best industries and Alpha Omega Ir-192 brachytherapy seeds. Med Phys 2004;31:3298.

4. Fippel M. A Monte Carlo dose calculation algorithm for proton therapy. Med Phys 2004;31:2263.

5. Allison J, Amako K, Apostolakis J, et al. GEANT4 developments and applications. IEEE Trans Nucl Sci 2006;53:270.

6. Hendricks JS, et al. MCNPX extensions, version 2.5.0. Los Alamos, NM: Los Alamos National Laboratory Report LA-UR-04-0570, 2004.

7. Seltzer SM. Electron-photon Monte Carlo calculations: The ETRAN code. Appl Radiat Isot 1991;42:917.

8. Online document at: http://physics.nist.gov/Phys RefData/Star/Text/ESTAR.html Accessed March 9, 2007.

9. Briesmeister JF. MCNPTM_A general Monte Carlo N-particle transport code, version 4B. Los Alamos, NM: Los Alamos National Laboratory Report LA-12625-M, 1997.
10. Poon E. Consistency test of the electron transport algorithm in GEANT4 Monte carlo code. Phys Med Biol 2005;50:681.

11. Schaart DR, Jansen JT, Zoetelief J, et al. A comparison of MCNP4C electron transport with ITS 3.0 and experiment at incident energies between 100 and $20 \mathrm{MeV}$ : Influence of voxel size, substeps, and energy indexing algorithm. Phys Med Biol 2002;47(9):1459.

12. Reynaert N, Palmans H, Thierens H, et al. Parameter dependence of the MCNP electron transport in determining dose distributions. Med Phys 2002;29 (10): 2446 .

13. Berger MJ. Monte Carlo calculation of the penetration of diffusion of fast charged particles. In: Alder B, Fernbach S, and Rotenberg M, eds., Methods in Computational Physics. New York: Academic Press, $1963 ; 135$.

14. Chakravarti L. Handbook of Methods of Applied Statistics, Vol. I. John Wiley \& Sons, 1967;392. 